\title{
Individual and Organizational Well-being when Workplace Conflicts are on the Agenda: A Mixed-methods Study
}

\section{Heidi Enehaug'}

PhD-student, Oslo and Akershus University College of Applied Sciences, Norway

I Migle Helmersen

PhD, Senior Researcher, Work Research Institute, Oslo and Akershus University College of Applied Sciences, Norway

\section{Svenn-Erik Mamelund}

PhD, Research Professor, Work Research Institute, Oslo and Akershus University College of Applied Sciences, Norway

\begin{abstract}
Previous studies have shown that direct involvement in workplace conflicts may have a significant impact on individual well-being. We used survey and interview data from a large nongovernmental organization (NGO) to analyze both the relationships between direct and indirect involvement in workplace conflicts and individual and organizational well-being. Results show that unaddressed conflicts and nonresponsive or conflict-involved managers are problematic because they fuel already existing conflicts, and also pave the way for new ones. If conflicts are not handled at an early enough stage, they seem to "paralyze" the organization and serve as an interlocking mechanism that contributes to hindering the necessary action from management. In our case, one-fifth of the employees were directly involved in the conflicts, and two-thirds felt that their local working environment had been influenced negatively by the conflicts. The prevalence of mental health problems in the NGO was almost twice as high as in the general Norwegian population, and slightly more than one out of 10 reported reduced work ability. We conclude that individuals directly involved in the conflicts experience negative health consequences, and that this fact, in combination with organizational issues and a very high share of employees indirectly involved in the conflicts, affected the well-being of the whole organization.
\end{abstract}

\section{KEY WORDS}

Direct and indirect conflict involvement / mastery / mental health / mixed methods / NGO / organizational well-being / social support / work ability / work environment

DOI

10.19154/njwls.v6il.4911

\section{Introduction and Background}

he literature on the association between direct involvement in interpersonal workplace conflicts and health has been growing in recent years. Direct involvement in interpersonal conflicts at work has been shown to be associated with individual mental health related outcomes such as insomnia (Sakurai et al., 2014), burnout

\footnotetext{
$\overline{{ }^{1} \text { E-mail: heidi.enehaug@hioa.no }}$
} 
(Fujiwara et al., 2003), depression (Inoue et al., 2010), psychiatric morbidity (Appelberg et al., 1991a; Romanov et al., 1996; Eriksen et al., 2006; Hyde \& Weathington, 2006), and organizational well-being outcomes such as job satisfaction, organizational commitment and turnover intentions (Frone, 2000), internal and external job mobility (Raeve et al., 2009),work engagement (Tsuno et al., 2009), and work disability (Appelberg et al., 1996).

In this article, we extend the prior literature on workplace conflicts by focusing both on individual and organizational mental well-being by distinguishing between those directly and indirectly involved in the conflicts. Whether horizontal (between co-workers) or vertical (worker-manager) in origin, we ask whether the consequences of conflicts go beyond the parties directly involved; if employees witnessing conflicts have a tendency to either try to withdraw themselves from the situation (and run the risk of being wrongfully positioned), or take sides in the conflicts, and as a consequence contribute to elevating and spreading the original conflict. In this article, we study workplace conflict and work-related health of employees in one large Norwegian Nongovernmental Organization (NGO, $N=294$ ). Rather than investigating only one indicator of work-related health, we focus on both mental health distress and work ability. Unlike most other studies on this topic, in addition to an analysis of quantitative survey data $(n=203)$ we also rely on qualitative interviews with more than one-fourth of the survey respondents $(n=82)$.

The overall aim of this article is to examine the importance of conflicts on an organizational level. We study (1) whether conflicts can jeopardize the well-being of a whole organization, (2) the relationships between direct and indirect conflict involvement and two self-reported health outcomes, and (3) whether the associations between direct and indirect conflict involvement and individual employee health are mediated by individual-level socio-demographic and psychosocial workplace variables?

\section{Data, methods, and analysis design}

\section{Study design}

Our study has a mixed-methods design, more specifically what has been labeled the exploratory sequential design (Creswell \& Clark, 2011). This implies that the qualitative data collection and analysis was prioritized in the first phase of the project, with the quantitative data collection and analysis in the second phase building on the results of phase one. The empirical material therefore consists of two sets of data: one derived from a large number of in-depth interviews and the other from a survey among all employees of the NGO.

This research and developmental project was initiated by an organization that for a period of several years had suffered from what key informants initially described as "a poor work environment, with many conflicts involving management and experiences that some employees found traumatic." Therefore, the project is problem oriented and should be regarded as applied research. This has important implications with regard to how the research process developed. There are various challenges concerning the need for anonymity. For some parts of the analysis, it was necessary to exclude (from this text) otherwise relevant and interesting information about the content of the conflicts 
uncovered. The need for anonymity also made it impossible to use several concrete quotes that easily could have identified the parties involved. The motivation for inviting the research team to help design a developmental process was first and foremost to address the ongoing internal troubles and the corresponding definitions of reality. The broad scope of the interview study was chosen so that as many employees as possible across organizational units would have the opportunity to express themselves. The idea was that this process would contribute to kick-start "the healing process" in the organization. The design of the research project was done in cooperation with an internal reference group, consisting of union officials, HR-personnel, management representatives, and the research team (five researchers). The reference group also functioned as key informants in the ongoing research process. This kind of design strategy has its roots within action research (see, e.g., Greenwood \& Levin, 2006), and "seeks to bring together action and reflection, theory and practice (...) in the pursuit of practical solutions to issues of pressing concern to people" (Savin-Baden \& Major, 2013, p. 245).

\section{Analysis model}

We use the mixed-method design to investigate (1) whether conflicts can jeopardize the well-being of a whole organization, (2) the relationships between direct and indirect conflict involvement and two self-reported health outcomes (work ability and symptoms of depression and anxiety), and (3) whether these associations were mediated by individual and organizational-level variables. Figure 1 presents the analytical model used to combine the datasets (survey and interviews) in our study with established knowledge concerning conflict involvement and selected individual and organizational-level factors.

Figure I: An analytical model of associations between conflict involvement, individual-level, and organizational-level factors based on current knowledge (solid arrows) and hypothesized relationships (dashed arrows).

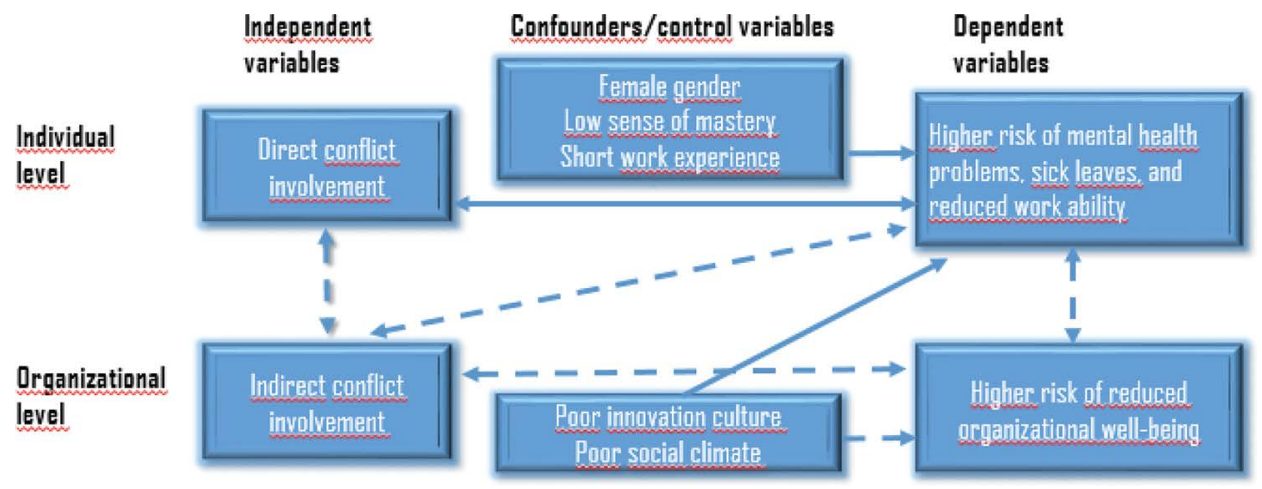

We suspect that the well-being of a whole organization can be affected when a large proportion of the organization is directly and indirectly involved in conflicts at the same time. We also expect that both direct and indirect conflict involvement is negatively associated with mental health problems (Fig. 1), even if being directly involved has more 
profound negative health effects. We also hypothesize that it is important to control for individual-level confounders such as female gender, low sense of mastery. and short work experience, as well as organizational-level confounders such as poor social climate and innovation culture, respectively, because-as discussed in upcoming sections-prior studies have demonstrated that these factors are negatively associated with a higher risk of experiencing symptoms of mental health problems. We further investigate whether the link between direct and indirect conflict involvement can work both ways. This would be the case if, for example, parties not directly involved in ongoing conflicts are pressured to choose sides, and as a consequence become unwilling participants in the conflict. Finally, we also acknowledge that the direction between conflict involvement and health can go both ways.

\section{Qualitative methods}

\section{Case description}

The NGO in question had 294 employees and could, by Norwegian standards, be categorized as a large company (more than 100 employees). The NGO is a decentralized organization consisting of several district offices, a main administrative unit, and locally based task-operating forces. In addition, the NGO has a volunteer membership organization that cooperates with the district offices and the task operating force and has its own local board. Finally, delegates from the local boards constitute a national congress with an advisory role to the NGO. The reputation of the NGO has traditionally been high in the Norwegian population. See Results section for further elaboration.

\section{Selection of informants}

The informants were selected from all parts of the NGO through a nomination process in the project's reference group. Each member of this group was asked to propose people on different sides of the known conflicts, and also to suggest people who were considered to be "neutral" in the main conflicts of the NGO. The persons with most nominations were asked to participate as informants in the study. All but two persons decided to be interviewed. See the Results section for description of conflicts.

\section{Interviews}

The interview study had three stated goals: first to contribute to addressing the conflicts of people directly involved in the conflicts and to start "the healing process" in the organization; second to give voice to large parts of the organization; and third to act as mean of data collection. More than one quarter $(28 \%)$ of the employees were interviewed over a period of 3 months (82/294). The interviews lasted an average duration of 1.5 to 2 hours. Focus group interviews were in some instances used to follow-up the individual interviews at the task operating forces in order to better understand the dynamics of the 
local work environment factors. The interviews focused on the future well-being of the organization and on informants' descriptions and opinions of how to facilitate positive change. Three of the main questions were: (1) How do you look upon the future if today's situation remains unchanged? (2) In your opinion, what are the essential areas of the whole organization in which improvements need to be made? (3) What changes are the most important for you in your daily work situation? In addition to these questions, the informants had the opportunity to address issues of specific interest to them and to give their version of the conflict-stories.

\section{Interview analysis}

The interviews were analyzed separately using a case study approach in order to map and "document multiple perspectives, while also acknowledging and presenting diverse points of view" (Savin-Baden \& Major, 2013, p. 163). Analysis of the transcribed interviews was conducted as a three-fold process: (1) singular researchers used margin notes and open coding to gain an overview of the content (Patton, 2002; Creswell, 2013), (2) margin notes and codes were discussed in the research group and broader categories/ themes were developed, and (3) the categories/themes then served as an input to the design of relevant questions and categories for the survey, as well as in the interpretation and analysis process of the study. Each informant quote in the Results section is numbered. We directly quote only a fraction of the 82 interviews, mainly as illustrative examples of the coding. The relatively low count of quotes should also be understood as a consequence of the informants' need for anonymity.

\section{Quantitative methods}

A questionnaire was sent to all employees in the NGO $(N=294)$. The response rate was high at $69 \%$, which gives a sample size of $n=203$. The Norwegian Social Science Data Services approved the study.

\section{Dependent variables}

The Hopkins Symptoms Checklist (HSCL-10) was used to assess mental health (Derogatis et al., 1974). The HSCL-10 is a short version of a more comprehensive test (HSCL25). However, the 10-question version performs just as well as the longer versions, and it has been psychometrically established both in population studies and in patient populations (Mouanoutoua \& Brown, 1995). In order to impose minimal time and resource demands upon the survey participants, we therefore chose HSCL-10 instead of HSCL25. Anxiety and depression are common stress-related disorders and are also closely related to illness behavior, such as seeking professional help, taking medication, and change in functioning (Sandanger et al., 1999). The 10 questions measuring the frequency and intensity of symptoms of anxiety and depression during the past week were scored on a scale from 1 (not bothered) to 4 (extremely bothered). The HSCL-10 total score was calculated as the sum score of items divided by the number of items answered. 
Respondents with an HSCL-10 score $\geq 1.85$ were considered a "case," that is, having symptoms of mental work related health problems (Winokur et al., 1982). The chosen cut-off of 1.85 is identical to standards used in previous workplace and population studies (Dalgard \& Lund Haheim, 1998). This permits the comparison of our results with other studies.

Work ability was assessed by the question "How do you estimate your work ability today?" This question was selected from the Grade Reduced Work Ability Scale, which was constructed for the Norwegian Ministry of Health and Social Affairs (Haldorsen et al., 1998). The responses were scored on a scale from 1 (extremely reduced) to 10 (not reduced at all). The work ability variable was recoded into two categories, the same way Haldorsen and colleagues did: reduced work ability (1-5) and not reduced work ability $(6-10)$. It has been found that self-evaluated work ability correlates significantly with clinically determined musculoskeletal capacity in healthy employees (Eskelinen et al., 1991). This provides some support for the construct validity of also using work ability in addition to mental health problems as a dependent variable in our study of the impact of conflict on health.

\section{Independent variables}

There appears to be no international consensus as to how to operationalize interpersonal workplace conflicts. Some use a dichotomous variable, asking the respondents questions such as "have you had considerable difficulties with superiors/co-workers" (Appelberg et al., 1991b) or "do you have conflicts with your co-workers/daily supervisors" (Raeve et al., 2009), while others have asked workers "how often do you get into arguments with others at work/with your supervisor?" (Frone, 2000). Oxenstierna (2011) asked about the source of the conflict, the importance of the conflict in addition to the dichotomous question "In the past two years, have you been drawn into any kind of conflict at your workplace?" Some have also used conflict scales developed for specific occupations (Fujiwara et al., 2003) or nations (Tsuno et al., 2009; Inoue et al., 2010; Sakurai et al., 2014).

All in all, conflict research seems to emphasize conflict direction and the magnitude of conflicts. In this study, we had the opportunity to combine two different datasets and thus gain a broader conception of what conflicts signifies. In the survey, we used two measures of conflict involvement-but no singular definition was provided. First, direct involvement in conflicts was measured by the question "Have you been directly involved in the conflicts?" Second, indirect involvement in conflicts was measured by the question "Do you experience that your local work environment has been negatively influenced by the conflict?" The answers were coded "yes" or "no." We also asked questions regarding where in the organization the informants would place the main conflict, whether the conflicts were case-oriented, person-oriented, or both, and whether managers provided any kind of support that helped them to cope with the conflicts. These questions were chosen as a direct result of the interview analysis that revealed three main conflicts and several smaller ones (see Results section). In the interview study, the informants elaborated on the content and the scope of the main conflicts, how they had been involved/ not involved in the conflicts, and also how they had experienced the situation and its consequences.

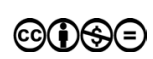




\section{Control variables}

We control for three individual-level variables and two organizational-level variables that may mediate the association between conflict and individual health or organizational well-being. The individual-level variables include gender, workplace experience, and mastery. Gender is an important confounder because females generally report a higher risk of anxiety and depression than males (e.g., Reneflot \& Mamelund, 2012: see also Fig. 1). Work experience was chosen on the basis of the assumption that extended seniority might have a soothing effect on conflict experiences. We also suspected that the longer seniority one had, the higher level of social support one might experience. Age was not assessed for anonymity reasons. The organizational-level variables include social climate and innovation culture. The latter control variables were chosen on the basis of the assumption that these might, one way or the other, influence the effects of the conflicts.

Mastery was the third of the individual-level variables and was assessed by three questions: "How often do you feel unsure about your task performance?", "How often do you experience situations where it is difficult to manage your tasks because of a deficit of time and resources?" and "How often do you need to manage work tasks despite the deficit of your qualifications?". Answers were scored 1 (Yes/often), 2 (Yes/ sometime), and 3 (No, rare/never). An index of these three questions was used in the analyses. Along with prior studies, the sense of mastery answers was dichotomized to insufficient mastery (yes/often and yes/sometimes) and sufficient mastery (No, rare/ never). Prior literature has also used mastery as a confounding control variable in their analyses of conflict and health (e.g., Raeve et al., 2009), but one could argue that mastery should be treated as an outcome variable along with work ability and other self-reported health variables. In this paper, however, we will treat mastery as a potential mediating variable because those with a low sense of mastery have been shown in prior research to suffer from higher levels of depression and anxiety (e.g., Reneflot \& Mamelund, 2012: see also Fig. 1).

Social climate was one of the organizational variables included in our quantitative analysis. It was assessed using three questions: The social climate at my workplace is (1) "encouraging and supporting," (2) "distrustful and suspicious," and (3) "relaxed and comfortable." Scores ranged from 1 (little or none) to 5 (very much). An index of these three questions was used in the analyses and answers were dichotomized to bad social climate (experience poor social climate/experience it sometimes) and good social climate (experience good social climate at the workplace). We dichotomize the social support variable in order to make our results comparable to prior studies that often apply the same dichotomizing. Another good reason to do this is the rather small sample size, which makes it less likely that statistically significant effects will be achieved when studying small subgroups. Low levels of social support are also positively related to depression and anxiety (e.g., Reneflot \& Mamelund, 2012: see also Fig. 1), and is therefore also an important control variable along with a low sense of mastery.

Innovation culture was the second organizational level variable and was assessed using the following three questions: (1) "Do employees take personal initiatives at your workplace?" (2) "Are employees encouraged to think about new ways of doing things at your workplace?" (3) "Is communication good enough at your department?" Scores ranged from 1 (very rare/never) to 5 (very often/all the time). An index of these three 
questions was used in the analyses and answers were dichotomized to bad innovation culture (experience bad innovation culture/experience it sometime) and good innovation culture (experience good innovation culture often/all the time) to increase the chance of obtaining statistically significant results. The three questions on innovation culture were included in the survey on the basis of the qualitative analysis that identified various perspectives on the importance and potential challenges connected to communication and innovation across and within organizational units. Innovation culture is moreover a potentially important control variable describing a pro-active attitude toward one's own work situation. Other research shows that insufficient communication and meager opportunities to take the initiative in ones work has a negative impact on job satisfaction (Elovainio et al., 2000). Along with this prior finding, we therefore hypothesize that poor innovation culture is associated with a higher risk of depression and anxiety and reduced level of work ability (Fig. 1). We further assume that a poor innovation culture negatively influence organizational well-being.

\section{Statistical methods}

A Chi-square test was used to analyze differences in proportions and Pearson's correlation was calculated for the independent and control variables. Logistic univariate models were used to examine the unadjusted association between, on the one hand, mental health and work ability, and the following variables on the other hand: direct and indirect involvement in conflicts, gender, sense of mastery, workplace experience, and the two psychosocial work environment variables, innovation culture and social climate. The final adjusted logistic multivariate regression model included only those control variables that were significant predictors of mental health problems or reduced work ability in the univariate analyses $(\mathrm{p}<0.05)$. Although the item "social climate" was significantly associated with both mental health and work ability in the univariate models, "social climate" was not included in the adjusted model (see Tab. 2, row 2) because it was highly correlated with the item "innovation culture" (0.6). A correlation coefficient of this magnitude may create a serious multi-collinearity problem. We decided to use the innovation culture variable in our further analyses rather than the social climate variable because the former index is more proactive, describing an active attitude toward one's own work situation. We used SAS, version 9.3, in order to do the statistical analyses.

\section{Validity and reliability}

This study has several strengths. The mixed-methods design has provided an extensive and in-depth understanding of the whole range of conflicts, tensions, and work environment issues of concern in this organization. Moreover, it has shed light on the prevalence of the variables and the possible associations between conflicts and individual health and organizational well-being. By combining the two different datasets, we were able to develop a broader conception of what conflicts means; the content and the scope of the main conflicts, who they involved or did not involve, how they developed and spread throughout the organization.

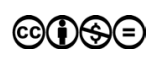


We have not identified previous studies on this topic that integrate quantitative and qualitative data and research methods. The qualitative study provided an understanding of conflict-development and evolution throughout the NGO that the survey data could not supply on its own terms. The survey data, on the other hand, provided means with which to measure associations between conflicts, work environment, mental health, and work ability using validated scores from work environment and health studies that would otherwise have been impossible to use. Furthermore, the survey data allow for more direct comparison of our results with those of other studies.

The data collection strategy and the data analysis strategy has increased the validity of the study in at least two ways: First, the number of interviews, the number of researchers (five) engaged in the study, and the chosen analysis strategy made it possible to control for researcher bias/personal stance and to increase reflexivity (see, e.g., Savin-Baden \& Major, 2013, pp. 68-83). The intended partial aim of the interview study-to kick start "the healing process" of the organization-through asking questions on a desired future-differed somewhat from the main aim of the survey study. The latter was focused on surveying the prevalence of the categories that stemmed from the qualitative analysis and to more directly address the possible association between work environment and health issues. Second, having the analytical possibility to interpret the final results simultaneously through the two main data sets made it easier to determine the truthfulness of the data, both ways. The high response rate $(69 \%)$ of the survey is another strength of our research. Because the data collection was carried out during an ongoing conflict, the risk of recall bias was reduced.

Our study also has limitations. First, although we have analyzed data for the whole population of the NGO, the quantitative analysis was made on a small number of individuals $(n=203)$. This constrains the opportunity to detect statistically significant effects. Second, we have not been able to ascertain how nonresponse may have affected our results because we have not had access to the population distributions on key demographic background variables. Third, our singular case approach may not be representative of other organizations that are subject to ongoing conflicts. Fourth, the cross-sectional design precludes our ability to draw conclusions regarding the direction of relationship among our study variables. Both anxiety and depression can affect the perception or experience of work stressors (Mills \& Mills, 2005) and hostility, life dissatisfaction, neuroticism, and stress of daily life have been shown to be associated with the risk of being involved in workplace conflicts (Appelberg et al., 1991b; Romanov et al., 1996). Some studies have shown that subjective appraisal of work conditions correlates more strongly with self-reported depression than objective work conditions (Goldberg et al., 1996). Moreover, it has been argued that the relation between work stress and depression may simply be attributable to underlying conflicts and career frustrations (Leventhal et al., 1996). It is important to note that the pathways linking psychosocial work conditions and mental health may not be direct, but bidirectional (see also Fig. 1). Thus, it cannot be precluded that the employees' mental state affected the report of psychosocial work conditions and the conflict dimensions. Fifth, the survey did not address whether the conflicts had a vertical (between co-workers) or horizontal (between managers and workers) origin, but chose to focus on organizational belonging. We nevertheless investigated these questions in-depth in the qualitative analysis and concluded that the NGO was characterized by serious vertical and horizontal conflicts. 


\section{Results}

We present our results in six steps. First, we present the results of the qualitative interview study along with a partial analysis. The latter was done in order to better present the choices made in the survey result section. This should also be regarded as an extensive case description. We stress that this part of the study was a predominant factor in determining which questions and variables to include in the survey. Some of the findings from the interview analysis have been left out of this part of the text in order to avoid reporting the same findings twice. We treat the whole of the result section as a more or less coherent "story" and discuss eventual conflicting findings across datasets later on. Second, we present the overall descriptive characteristics of the conflicts, as well as the descriptive characteristics of the dependent, independent, and control variables used in the quantitative analysis. We also report on the two dependent variables broken down by gender and workplace experience. Third, we present descriptive characteristics of the respondent's conflict involvement (directly and indirectly) by the dependent, independent, and control variables. Fourth, we present the results from bivariate correlation analysis. In the fifth and sixth steps, we present the univariate and multivariate results.

\section{Main results from the interview study}

For a long period prior to the data collection taking place, the NGO was subject to extensive and negative publicity whereby internal affairs and conflict issues were addressed on a regular basis. Organizational members on different levels used the media in order to present their views. Members of the leadership group resigned. Judicial steps were also taken in order to remove organizational members. The NGOs' traditionally good reputation was subject to negative change and the organization was characterized by serious vertical and horizontal conflicts.

The overall qualitative analysis revealed a working environment characterized by deeply rooted tensions, full blown conflicts, and trust deficiencies. These problems, in general, originated in structural organizational traits, in insufficient or counterproductive cooperation between employees and units, and in poor management and communication practices within the NGO. A lot of these controversies had probably been in the air for a long period of time, but grew in strength and scope because they were not handled in an appropriate manner.

We identified three interrelated main conflicts in the NGO. Communication and cooperation between management and employees within the headquarters (administrative units) suffered severely and developed into the first main conflict. The situation worsened because some of the leaders were directly involved in the second main conflict (see below) or from managers' avoidance strategies' and their lack of problemsolving capabilities. This, in turn, contributed to a serious lack of trust both vertically and horizontally. Furthermore, the interviewees reported that the decrease in social support became problematic. The following informant statement is typical for this situation:

We have not been supportive enough of each other, we have not taken care of one another, and setting the right limits and knowing what is wrong and what is right (no 31) 
Both the employees and the leaders were polarized in views regarding leadership style, future organizational structure, and issues regarding the usefulness of different income sources. What appeared to be conflicts of interest soon turned into full-blown horizontal and vertical personified conflicts. The leaders were perceived to not only be parties in the conflicts but also to be the "root of the problems" by many of the employees. Some of the leaders who had been drawn into the conflicts experienced a strong sense of guilt because of the way they had acted. One of them said:

I have been part of destroying the lives of many of my colleagues who had to quit their jobs because of my actions. If I had given it more thought, I should not have been involved, but should have tried to stop the negative situation developing instead (no 67)

This quote offers an example of the interlocking effect that unaddressed conflicts may have on the well-being of an organization. The inability or unwillingness to handle tensions and disagreements may act as an accelerant in a conflict development process. The situation gradually extends in scope and seriousness. If conflicts are not dealt with at an early enough stage, they seem to "paralyze" the organization and serve as an interlocking mechanism that contributes to hindering the taking of necessary action from management. This process further seems to boost the personification of the conflict. In addition, the data indicate an association between the personification of conflicts and the ability to describe the conflict origin. This has at least two possible alternative explanations: that when the personification of conflicts sets in, the conflict origin definition is blurred, or that, when conflicts have been going on unaddressed long enough, the understanding of the origin fades into the background, and persons or conflict parties, come to the forefront.

The second main conflict came to the fore when the (former) top leader, a charismatic but allegedly controlling figure through several dispositions and actions managed to cast the organization into a state of despair. Allegations of bullying from this leader and organizational members that were described as "followers" forced employees and managers to choose sides in the conflicts:

During the reign of the top leader, the work environment was impossible to cope with. You were either for or against. You did not know who to trust. There was a feeling of lack of safety and fear in the organization (no 33)

A feeling of distrust, insecurity, and various kinds of rumors thus spread throughout the organization. At the time of the data collection, these tensions and conflicts were not resolved. One of the employees described the escalation of the situation:

There has been a very high level of conflict for a long period of time. You are drawn into the conflicts against your own will. The flow of rumors is frightening. We should focus on bettering our work environment. In the end, you get psychological trouble (no 3)

Another finding was that even if the various units of the organization were interdependent, they partly lived a life of their own-in a (too) loosely coupled system. This in turn can explain some communication problems, misinterpretations, and attitudes of skepticism toward "the others" actions. 
The third main conflict-the unaddressed conflict over resources between (the volunteer organization) the task operating forces and the administrative staff at headquarters regarding economical allocations, decision making (or the lack of it), communication channels, and the lack of meeting spaces, was a frequent topic in the interviews. Members of the task operating forces defined themselves as situated within the heart of the organization, they performed the job the NGO was set up to do, and the volunteer part of the organization helped make this possible through their combined efforts to provide small and large practical or economical contributions. The volunteer organization expressed similar concerns, especially concerning economic prioritization within the NGO. The administrative units were to a larger extent thought to be resource spenders. We interpret this as an issue concerning the power to define the main goal of the organization: the closer the interviewees were to "the true organizational goal," the more morally inclined they felt to the "truth." We also see this as an expression of the lack of knowledge, understanding, and positive images of the content and performance of the work of the administrative unit.

Whether or not directly involved in conflicts, the vast majority of the interviewees reported that they felt pressured or obliged to "take sides" in one or several of the ongoing main-or smaller conflicts. This situation put strain on all parties with some describing this process as detrimental both to their mental health and to their work ability. Some describe withdrawal tendencies or physical stressors, some underline exhaustion, while yet others have lost their sense of job satisfaction or have experienced reduced energy levels in general. The five quotes below illuminate various perspectives on how conflicts may have an effect on an individual level.

I have been in the middle of the conflicts. It affects you. You are exhausted by everything you talk about it (no 23)

It is difficult to explain the situation to my family and friends. It is unreal (...) I am constantly walking around with a knot in my chest, and I do not sleep well (no 17)

It really affected my health. I was afraid to go to work for a period of time (no 4)

It feels hard to see that people have been disloyal and that they can push others without being affected themselves. This eats energy.(..) Local interests inhibit putting the best interest of everyone first. It is a very negative situation for colleagues (no 73)

You get devoured by negativity when you come to work. (...) There are some strong negative forces (..) very little respect for what others do; you do not really know what your colleagues are up to. (...) I am struggling now, and I do not know if I want to go on. That is a shame. I do not like to leave things half-done but I have to consider my own health. I don't look forward to going to work (no 50)

\section{Qualitative summary remarks}

The qualitative organizational analysis demonstrates an organization with multiple conflicts and tensions, which produced trouble both from an individual and organizational point of view (Fig. 2). The major concern was the limited competence or willingness 
Figure 2: Conflict development model.

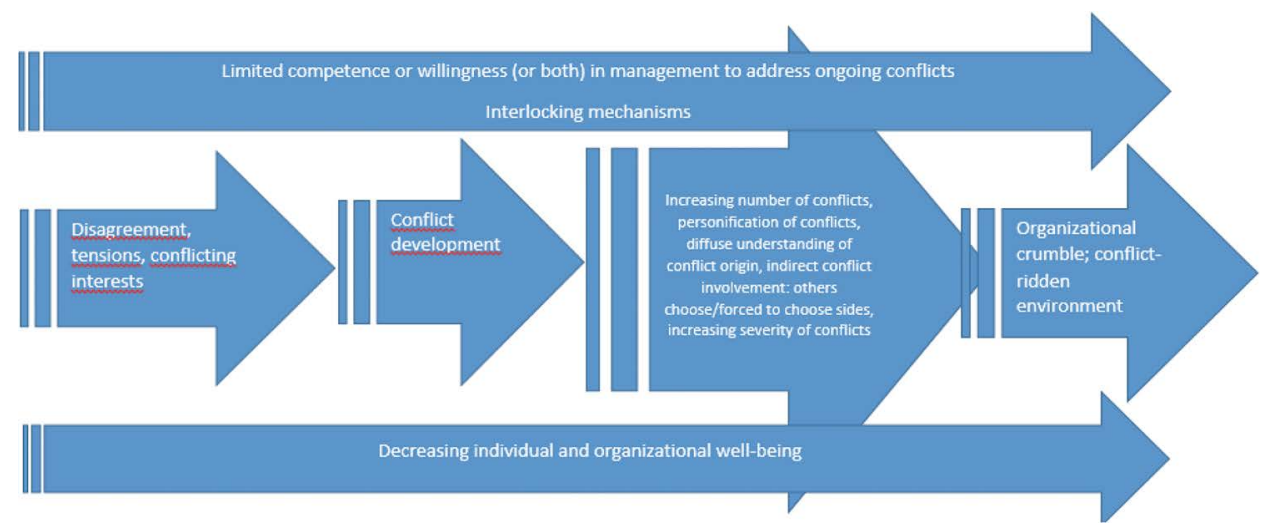

(or both) to address ongoing conflicts, especially on the management level. Unaddressed conflicts and nonresponsive or conflict-involved managers are cause of concern because they seem to feed the conflicts already there, and also to pave the way for new ones. The organization could best be described as in a state of crumble.

With this in mind, we turn our attention to what the survey material revealed, before we discuss and offer further conclusions.

\section{Descriptive characteristics in the survey material}

\section{Dependent variables}

We have analyzed two dependent variables, work ability and mental health. All survey respondents answered the work ability question. Of the 203 responding employees, $12 \%$ experienced reduced work ability (Tab. 1 ). The mean score on the work ability index was 7.7 (where 1 was extremely reduced, 10 was not reduced at all). The two groups (reduced or not reduced work ability) did not statistically differ in gender or work experience (results not shown).

A total of 196 employees completed the HSCL-10 questions tapping mental health. The mean score was 1.3 , with $17 \%$ (33 of 196) reporting an HSCL-10 score $\geq 1.85$ (Tab. 1). Women reported more symptoms of depression and anxiety than men (32 vs. $14 \%$ ). Employees with relatively short workplace experience, between 2 and 5 years, reported the most mental health problems (30\%) (results not shown). The gender and seniority differences were statistically significant and in accordance with our expectations (Fig. 1).

\section{Independent variables}

One in five of the survey respondents reported to be directly involved in the conflicts and two-thirds felt that their local work environment had been negatively influenced by 
Table I Descriptive characteristics of respondent's conflict involvement by dependent, independent, and confounding variables, in \% $(n)^{*}$

\begin{tabular}{|c|c|c|c|c|c|c|}
\hline & & \multicolumn{2}{|c|}{$\begin{array}{l}\text { Directly involved } \\
\text { in conflicts } \\
\%(n)\end{array}$} & \multicolumn{2}{|c|}{$\begin{array}{l}\text { Indirectly involved } \\
\text { in conflicts } \\
\%(n)\end{array}$} & \multirow[t]{2}{*}{$\begin{array}{c}\text { Total } \\
\text { sample } \\
\%(n)\end{array}$} \\
\hline & & Yes & No & Yes & No & \\
\hline & $\begin{array}{l}\text { Total sample } \\
\% \text { (n) }\end{array}$ & $21(42)$ & $79(160)$ & $67(136)$ & $31(63)$ & 100 (203) \\
\hline \multirow{2}{*}{$\begin{array}{l}\text { Reduced work } \\
\text { ability }\end{array}$} & Yes & $24(10)$ & $9(14)$ & $15(20)$ & $6(4)$ & $12(24)$ \\
\hline & No & $76(32)$ & $91(146)$ & $85(116)$ & $94(59$ & $88(179)$ \\
\hline \multirow{2}{*}{$\begin{array}{l}\text { Mental health } \\
\text { problems }\end{array}$} & Yes & $37(15)$ & $12(18)$ & $23(30)$ & $5(3)$ & $17(33)$ \\
\hline & No & $63(26)$ & $88(136)$ & $77(102)$ & $95(57)$ & $83(163)$ \\
\hline \multirow[t]{3}{*}{ Gender } & Men & $71(30)$ & $86(137)$ & $79(108)$ & $89(56)$ & $82(167)$ \\
\hline & Women & $26(11)$ & $14(23)$ & $20(27)$ & I। (7) & $17(35)$ \\
\hline & Missing & $2(1)$ & - & I (I) & - & $0.5(1)$ \\
\hline \multirow{4}{*}{$\begin{array}{l}\text { Work experience } \\
\text { (years) }\end{array}$} & $<2$ & $12(5)$ & $26(4 I)$ & $21(28)$ & $27(17)$ & $23(46)$ \\
\hline & $2-5$ & $10(4)$ & $15(24)$ & $15(2 \mid)$ & I। (7) & $14(28)$ \\
\hline & $5-10$ & $26(11)$ & $28(44)$ & $29(40)$ & $24(15)$ & $28(56)$ \\
\hline & $10-20$ & $52(22)$ & $32(5 \mathrm{l})$ & $35(47)$ & $38(24)$ & $36(73)$ \\
\hline \multirow[t]{3}{*}{ Social climate } & Good & $38(16)$ & $61(97)$ & $50(68)$ & $68(43)$ & $55(113)$ \\
\hline & Bad & $55(23)$ & $38(60)$ & $48(65)$ & $27(17)$ & $4 \mid(84)$ \\
\hline & Missing & $7(3)$ & $2(3)$ & $2(3)$ & $5(3)$ & $3(6)$ \\
\hline \multirow{3}{*}{$\begin{array}{l}\text { Innovation } \\
\text { culture }\end{array}$} & Good & $41(17)$ & $63(100)$ & $53(72)$ & $67(42)$ & $58(117)$ \\
\hline & Bad & $50(21)$ & $34(55)$ & $43(59)$ & $27(17)$ & $38(77)$ \\
\hline & Missing & $10(4)$ & $3(5)$ & $4(5)$ & $6(4)$ & $4(9)$ \\
\hline \multirow[t]{2}{*}{ Mastery } & Sufficient & $26(11)$ & $53(84)$ & $40(55)$ & $62(39)$ & $47(96)$ \\
\hline & Insufficient & $74(31)$ & $48(76)$ & $60(8 I)$ & $38(24)$ & $53(107)$ \\
\hline
\end{tabular}

*Respondents with missing values on mental health problems $(n=7)$, missing values on direct conflict involvement $(n=1)$, and missing values on indirect conflict involvement $(n=4)$ are not displayed in this table.

the conflicts (Tab. 1). We asked the respondents who had been affected personally by the conflicts whether they regarded the conflicts to be case-oriented, person-oriented, or both person-oriented and case-oriented. We found that nobody regarded the conflicts to be purely case-oriented. Thirty-six percent regarded the conflicts to be purely personoriented. Thirty-eight percent meant that the conflicts were both person-oriented and case-oriented, while $26 \%$ did not answer this question. 


\section{Control variables}

More than eight out of 10 of the survey respondents were men (Tab. 1). The sample had differing seniority: $23 \%$ had worked for less than 2 years in this particular NGO, $14 \%$ between 2 and 5 years, $28 \%$ between 5 and 10 years, and $36 \%$ between 10 and 20 years.

The social climate at work was considered good by more than half of our respondents. Almost six out of 10 found that the innovation culture was good. With regard to mastery in the work situation, $47 \%$ reported sufficient mastery and $53 \%$ of the respondents reported insufficient mastery.

\section{Conflict involvement by dependent, independent, and confounder variables}

The results in Table 1 clearly show that those who were directly or indirectly involved in the conflicts have a larger proportion with reduced work ability and a higher proportion with symptoms of mental health problems than those not involved in either way. It is clear that those directly involved were more affected than the indirectly involved.

Table 1 also shows that a slightly higher proportion of women than men were involved in the conflicts (directly involved). We also see that those directly involved had longer seniority (in particular those with 10-20 years seniority), while an indirect involvement was more equally distributed among workers with various years of work experience. Further, the social climate was poorer among those directly involved than those who were not directly involved. We see similar results for those indirectly involved versus those not indirectly involved, but both groups of not involved have a lower proportion with bad social climate than the corresponding groups of directly involved in the conflicts. Finally, we see that among those directly involved, the proportion with a poor innovation culture and insufficient mastery is much higher than for those not directly involved. For those indirectly involved, we see the same pattern as previously described for social climate.

\section{Correlation analysis}

There were some small positive but significant correlations between the psychosocial work environment and conflicts variables: social climate and mastery (0.2); social climate and directly involving in the conflicts $(0.2)$; social climate and experience "that local work environment has been influenced by the conflict indirectly" (0.2). The item social climate correlated highly $(0.6)$ and significantly with innovation culture $(\mathrm{p}<0.01)$. To avoid a multicollinearity problem, we therefore only included innovation culture in our multivariate models.

\section{Univariate logistic regression}

Table 2 shows the unadjusted and adjusted odds ratios for the associations between the independent and control variables and the risk of reporting reduced work ability and having elevated HSCL-10 score (symptoms of mental health problems). Here, we first comment on the unadjusted results: 
Table II Logistic relationship between reduced work ability, mental health problems, and conflict involvement, demographic and personal, psychosocial work environment variables, and conflict variables among respondents working in a Norwegian NGO*

\begin{tabular}{|c|c|c|c|c|c|c|c|c|}
\hline \multirow[b]{3}{*}{ Risk factors } & \multicolumn{4}{|c|}{ Reduced work ability $(n=203)$} & \multicolumn{4}{|c|}{ Mental health problems $(n=196)$} \\
\hline & \multicolumn{3}{|c|}{ Unadjusted } & \multirow{2}{*}{$\begin{array}{c}\text { Adjusted } \\
95 \% \mathrm{Cl}\end{array}$} & \multicolumn{3}{|c|}{ Unadjusted } & \multirow{2}{*}{$\begin{array}{c}\text { Adjusted } \\
95 \% \mathrm{Cl}\end{array}$} \\
\hline & OR & $95 \% \mathrm{Cl}$ & OR & & OR & $95 \% \mathrm{Cl}$ & OR & \\
\hline \multicolumn{9}{|c|}{$\begin{array}{l}\text { Directly involved } \\
\text { in the conflicts }\end{array}$} \\
\hline No (ref.) & 1.0 & Ref. & 1.0 & Ref. & 1.0 & Ref. & 1.0 & Ref. \\
\hline Yes & 3.3 & $1.3-8.0$ & 2.6 & $0.9-7.0$ & 4.4 & $2.0-9.7$ & 3.5 & $1.3-9.5$ \\
\hline
\end{tabular}

Indirectly involved in the conflicts

\begin{tabular}{lllllllll} 
No (ref.) & 1.0 & Ref. & - & - & 1.0 & Ref. & 1.0 & Ref. \\
Yes & 2.5 & $0.8-7.8$ & - & - & $\mathbf{5 . 6}$ & $\mathbf{I . 6 - 1 9 . 1}$ & 3.5 & $0.9-12.9$ \\
\hline
\end{tabular}

\section{Gender}

\begin{tabular}{lllllllll} 
Men (ref.) & 1.0 & Ref. & - & - & 1.0 & Ref. & I.0 & Ref. \\
Women & 1.3 & $0.5-3.7$ & - & - & $\mathbf{3 . 0}$ & $\mathbf{I . 3 - 7 . 1}$ & 2.7 & $0.9-12.9$ \\
\hline
\end{tabular}

\section{Work experience} (years)

$\begin{array}{lllllllll}<2 \text { (ref.) } & 1.0 & \text { Ref. } & 1.0 & \text { Ref. } & 1.0 & \text { Ref. } & 1.0 & \text { Ref. } \\ 2-5 & 2.6 & 0.4-16.9 & 2.6 & 0.4-17.8 & \mathbf{5 . 9} & \mathbf{I . 4 - 2 4 . 7} & \mathbf{5 . 7} & \mathbf{I . 2 - 2 8 . 0} \\ 5-10 & \mathbf{4 . 8} & \mathbf{I . 0 - 2 3 . 1} & 3.5 & 0.7-18.2 & \mathbf{4 . 1} & \mathbf{I . I - 1 5 . 6} & 3.1 & 0.7-13.4 \\ 10-20 & 3.1 & 0.6-15.0 & 1.9 & 0.4-9.8 & 2.3 & 0.6-8.8 & 1.5 & 0.3-6.9\end{array}$

\section{Social climate}

\begin{tabular}{llllllll} 
Good (ref.) & 1.0 & Ref. & - & - & 1.0 & Ref. & - \\
Bad & $\mathbf{2 . 5}$ & $\mathbf{1 . 0 - 6 . 1}$ & - & - & $\mathbf{3 . 9}$ & $\mathbf{1 . 7 - 8 . 8}$ & - \\
\hline
\end{tabular}

\section{Innovation culture}

\begin{tabular}{|c|c|c|c|c|c|c|c|c|}
\hline Good (ref.) & 1.0 & Ref. & 1.0 & Ref. & 1.0 & Ref. & 1.0 & Ref. \\
\hline Bad & 6.3 & $2.2-|8|$. & 5.5 & $|.9-| 6 . \mid$ & 4. 1 & I.8-9.4 & 3.7 & 1.4-9.6 \\
\hline \multicolumn{9}{|l|}{ Mastery } \\
\hline Sufficient (ref.) & 1.0 & Ref. & - & - & 1.0 & Ref. & 1.0 & Ref. \\
\hline Insufficient & 1.9 & $0.8-4.7$ & - & - & 4.3 & $1.7-10.3$ & 2.6 & $0.9-7.0$ \\
\hline
\end{tabular}

*Bold figures are significant at 5\% level.

\section{Direct conflict involvement influences mental health problems and work ability negatively}

Those who were directly involved in the conflicts had a significantly higher risk of reporting both reduced work ability and mental health problems. The experience that the local work environment had been influenced by the conflict indirectly, however, was significantly related only to symptoms of mental health problems. 


\section{Gender and work experience influence mental health}

The univariate analysis also demonstrated that women were significantly associated with symptoms of mental health problems as expected, but not with reduced work ability (Fig. 1). Seniority between 5 and 10 years appeared to be associated with reduced work ability. Even less experienced employee groups (2-5 years) and the group with 5-10 years seniority had significant associations with symptoms of mental health problems.

\section{Poor social climate and innovation culture affects both mental health and work ability in a negative way}

As expected, those employees who reported poor social climate and bad innovation culture had a significantly greater risk of reporting poor work ability and mental health problems (Fig. 1). Contrary to our expectations, we found no significant associations between insufficient mastery and reduced work ability in the univariate model. Nevertheless, those who reported insufficient mastery had a significantly higher risk of reporting symptoms of mental health problems in the univariate model. This latter finding is thus in line with our expectations (Fig. 1).

\section{Multiple logistic regression}

Table 2 also shows the adjusted models. In the multivariate regression models, we only included the variables that had significant elevated incidence ratios in the univariate analyses. In the multivariate analysis, poor innovation culture was the only variable that was significantly related to insufficient work ability. Work experience between 2 and 5 years, poor innovation culture, and direct involvement in the conflicts remained significant for symptoms of mental health problems.

\section{Discussion}

We have documented a high prevalence of both direct and indirect conflict involvement, and of individual mental health problems in our case, along with dysfunctional mechanisms in the working environment that boosted the magnitude and scope of the conflicts. The magnitude of conflict involvement and health-related issues is indeed much higher than for other comparable study samples. This detrimental combination jeopardized the well-being of the whole organization. Our results also illustrated several key distinguishing psychosocial, conflict, and demographic characteristics, which significantly influenced work ability and mental health. We found that reduced work ability was independently associated with innovation culture. Mental health problems were independently associated with seniority and innovation culture and direct involvement in the conflicts.

\section{Conflict prevalence, individual health, and work ability}

Our respondents' score is above average of comparable working groups both in relation to the level of conflicts, mental health issues, and sickness absence rates. First, one 
in five were directly involved in the conflicts and two-thirds felt that their local work environment suffered because of the conflicts. A comparison with a sample survey of the general Norwegian working population shows that the proportion "often" involved in interpersonal conflicts with superiors or co-workers was $8.5 \%$ and $2.8 \%$, respectively (https://www.ssb.no/statistikkbanken). Two European studies found that such conflicts occurred for 7-9\% (Appelberg et al., 1991b; Raeve et al., 2009). This indicates that the proportion of workers directly involved in conflicts in our sample was up to three times more frequent than in these comparison groups $(21 \%$ vs. $7 \%)$.

Second, approximately $12 \%$ of the workers in our study reported reduced work ability and $17 \%$ reported symptoms of depression and anxiety. We have not found comparable figures on work ability from other data sources or studies, but the prevalence of mental health problems in the NGO was almost twice as that in the general Norwegian population (Reneflot \& Mamelund, 2012). Third, although we were not able to study the individual-level association between conflict involvement and sickness absence rates, our study documented that the average sickness absence rates in the various units of the NGO ranged between $6 \%$ and $8 \%$. The average national sickness absence rates were $5 \%$ for men and $8 \%$ for women. Although eight out of 10 people in the NGO were men (Tab. 1), their sickness absence rates were more similar to the female than the male national average. The higher than expected level of mental health problems and sickness absence rates, in combination with the high number of people directly and indirectly involved in the conflicts, in addition to the internal organizational difficulties, together support our hypothesis that "organizational well-being" is affected.

\section{Direct and indirect conflict involvement vs. mental health and work ability}

Direct conflict involvement had a strong and independent association with symptoms of mental health problems but not with reduced work ability. Our results regarding the association between direct conflict involvement and symptoms of depression and anxiety are in line with previous research on conflict and employee mental health (Appelberg et al., 1991b; Romanov et al., 1996; Eriksen et al., 2006; Hyde \& Weathington, 2006; Inoue et al., 2010). To our knowledge, prior studies did not analyze the association between conflict and work ability. We found that direct involvement in conflicts was positively associated with reduced work ability in the univariate model, but after controlling for work experience and innovation culture, the association was no longer significant. One possible explanation for this may be that our cut-off point was too low (cut-off were between 5 and 6 on a scale from 0 to 10). Another possible explanation is a low sample size, which makes it more difficult to document significant effects (the rather large confidence intervals for several of the estimates are an indication of this problem). A third possible explanation is that the question used to assess work ability could be interpreted in many ways because of the general phrasing of it. Perhaps work ability scores should be based on a more subtle series of questions, instead of the one singular (thus unprecise) question?

On the basis of the qualitative analysis, we expected that the conflict-ridden local work environments of the NGO would have an effect on both work ability and the mental health of the employees. Results showed that such work environments had an effect only on mental health in the univariate analyses and that this effect vanished in the 
adjusted logistic multivariate regression. There was also an unadjusted elevated risk of reduced work ability when informants reported to be indirectly involved, but this association did not reach the significance level set at $5 \%$. There are several reasons for this (see the above section). The interview material shows that even though the informants were not directly involved, they still felt unease, despair, and, to a certain extent, exhaustion as a result of the conflicts and general distrust in the organization. Nevertheless, our findings show that direct conflict involvement is a stronger risk factor to mental health problems than indirect conflict involvement.

Less involvement in a conflict increases the chance that a worker can perform work tasks regardless of what is going on (see Tab. 1). Among the respondents who had been affected personally by the conflicts, we also found that three-quarters regarded the conflicts to be purely person-oriented or both person-oriented and case-oriented. These results illustrate that many of the conflicts of this particular NGO were personified conflicts. The qualitative data describe this in further detail, suggesting that even if conflicts started out as tensions or conflicts of interests, resources, or information (in our material the equivalent to case-oriented), they often ended up as personified conflicts. One possible explanation for the escalation of the conflicts is that the leaders were too involved in the conflict to serve as conflict-managers.

\section{Innovation culture versus work ability and mental health}

Poor innovation culture had a strong influence on work ability and mental health in our study (Tab. 2). This finding is in line with prior research (Elovainio et al., 2000). The qualitative analysis revealed that the NGO could best be described as a conflictridden organization, partly because of the poorly functioning organization structure and the insufficient cooperation and understanding between different units and layers of the NGO. Innovation culture was therefore, as expected, also an important mediating confounder to take into account in our study (Fig. 1). The interviews also revealed that the task operating force in the organization felt a strong irritation and a feeling of being distrusted by the administrative units and the centrally placed leaders. This was because they had what was described as both poor and insufficient communication channels with the administrative units. They also reported that initiatives rarely resulted in concrete changes, and many of them felt that they were subject to ridicule or that they were not taken seriously. The administrative units were less critical of the task operating forces than the other way around-see interview analysis section. In addition, employees in other parts of the organization gave extensive examples of poor communication and communicated a feeling that it was pointless to take initiative because it did not lead to changes.

\section{Conclusion}

By doing a mixed-methods study, we were able to identify the conflict development process in this organization. This further provided a "thick analysis" (Clarke, 2005) of the whole range of potential effects conflicts may have on individual and organizational well-being. This study has revealed that conflicts can be about almost anything, 
between all parties in an organization and that it affects not only those directly involved but also the circles around. Unaddressed conflicts, nonresponsive or conflict-involved managers raise concern because they may feed current conflicts, and also pave the way for new ones. The added value of this study is the focus on the propagation of conflict involvement and of organizational factors. We show that it is not only the parties directly involved in conflicts, but also those indirectly involved, who experience the consequences of strongly personified conflicts. Moving from direct (one-fifth) to indirect conflict involvement (two-thirds), we see a dramatic increase in people affected by conflicts. Our results suggest that unaddressed conflicts have a significant effect on the overall well-being of an organization and that conflict management should be an important part of organizational policies.

The survey analysis showed that the conflicts had a less negative impact on the employees' work ability than we initially expected. There was a significant association between direct conflict involvement and reduced work ability in the bivariate model. However, when controlled for work experience and innovation culture, this association disappeared. Indirect conflict involvement was not related to reduced work ability in either model. It is an interesting finding that the employees in our case were actually able and willing to continue working throughout these difficult times. The qualitative findings revealed that the overall level of social support declined during the conflicts. One reason why the employees kept up their work ability, nevertheless, may be that some local pockets in the organization managed to continue providing sufficient social support and that this acted as a buffer mechanism. Local parts of an organization more willing to facilitate an innovative culture may also have a buffering function that makes coping with detrimental effects of conflicts easier.

We found that direct involvement in conflicts was a predictor of mental health problems, but there was no independent association between indirect conflict involvement and mental health. Poor innovation culture negatively affects both the work ability and the mental health situation of the employees. Employees with a work experience of 2-5 years were more prone to develop mental health problems.

We suggest that further research would benefit from (1) utilizing a mixed-methods approach, and (2) that measures are taken to develop an index for conflict origin, conflict involvement, and possible organizational and health outcomes linked to conflict. This could potentially enhance the possibility to perform cross-national and crosssectional studies that actually investigate the same phenomena.

\section{References}

Appelberg K, Romanov K, Heikkila K, et al. (1996) Interpersonal conflict as a predictor of work disability: a follow-up study of 15,348 Finnish employees. J Psychosom Res 40: 157-167. doi: http://dx.doi.org/10.1016/0022-3999(95)00576-5.

Appelberg K, Romanov K, Honkasalo M-L, et al. (1991) Interpersonal conflicts at work and psychosocial characteristics of employees. Soc Sci Med 32: 1051-1056. doi: http://dx.doi.org/10.1016/0277-9536(91)90162-6.

Clarke A. (2005) Situational Analysis: Grounded Theory After the Postmodern Turn. London: Sage.

Creswell JW. (2013) Qualitative Inquiry \& Research Design. Chosing among five approaches. Los Angeles: Sage.

\section{(ㄷ)(1) $\Theta$}


Creswell JW and Clark PLV. (2011) Designing and Conducting Mixed Methods Research. California: Sage Publications, Inc.

Dalgard OS and Lund Haheim L. (1998) Psychosocial risk factors and mortality: a prospective study with special focus on social support, social participation, and locus of control in Norway. J Epidemiol Community Health 52: 476-481. doi: http://dx.doi.org/10.1136/jech.52.8.476.

Derogatis LR, Lipman RS, Rickels K, et al. (1974) The Hopkins Symptom Checklist (HSCL). A measure of primary symptom dimensions. Mod Probl Pharmacopsychiatry 7: 79-110. doi: http://dx.doi.org/10.1159/000395070.

Elovainio M, Kivimaki M, Steen N, et al. (2000) Organizational and individual factors affecting mental health and job satisfaction: a multilevel analysis of job control and personality. J Occup Health Psychol 5: 269-277. doi: http://dx.doi.org/10.1037/1076-8998.5.2.269.

Eriksen W, Tambs K and Knardahl S. (2006) Work factors and psychological distress in nurses' aides: a prospective cohort study. BMC Public Health 6: 290. doi: http://dx.doi.org/10.1186/1471-2458-6-290.

Eskelinen L, Kohvakka A, Merisalo T, et al. (1991) Relationship between the self-assessment and clinical assessment of health status and work ability. Scand J Work Environ Health 17 Suppl 1: 40-47.

Frone MR. (2000) Interpersonal conflict at work and psychological outcomes: testing a model among young workers. Journal of Occupational Health Psychology 5: 246. doi: http://dx.doi.org/10.1037/1076-8998.5.2.246.

Fujiwara K, Tsukishima E, Tsutsumi A, et al. (2003) Interpersonal conflict, social support, and burnout among home care workers in Japan. J Occup Health 45: 313-320. doi: http://dx.doi.org/10.1539/joh.45.313.

Goldberg P, David S, Landre MF, et al. (1996) Work conditions and mental health among prison staff in France. Scand J Work Environ Health 22: 45-54. doi: http://dx.doi. org/10.5271/sjweh.108.

Greenwood DJ and Levin M. (2006) Introduction to Action Research: Social Research for Social Change. California: SAGE publications.

Haldorsen EM, Indahl A and Ursin H. (1998) Patients with low back pain not returning to work. A 12-month follow-up study. Spine 23: 1202-1207; discussion 1208. doi: http://dx.doi.org/10.1097/00007632-199806010-00004.

Hyde RE and Weathington BL. (2006) The congruence of personal life values and work attitudes. Genet Soc General Psychol Monographs 132: 151-190. doi: http://dx.doi.org/ 10.3200/mono.132.2.151-192.

Inoue A, Kawakami N and Group HCS. (2010) Interpersonal conflict and depression among Japanese workers with high or low socioeconomic status: findings from the Japan Work Stress and Health Cohort Study. Soc Sci Med 71: 173-180. doi: http://dx.doi.org/10.1016/j.socscimed.2010.02.047.

Leventhal E, Hansell S, Diefenbach M, et al. (1996) Negative affect and self-report of physical symptoms: two longitudinal studies of older adults. Health Psychol 15: 193-199. doi: http://dx.doi.org/10.1037/0278-6133.15.3.193.

Mills LD and Mills TJ. (2005) Symptoms of post-traumatic stress disorder among emergency medicine residents. J Emerg Med 28: 1-4. doi: http://dx.doi.org/10.1016/j. jemermed.2004.05.009.

Mouanoutoua VL and Brown LG. (1995) Hopkins Symptom Checklist-25, Hmong version: a screening instrument for psychological distress. J Person Assess 64: 376-383. doi: http://dx.doi.org/10.1207/s15327752jpa6402 16.

Patton MQ. (2002) Qualitative research \& evaluation methods. Third Edition. London: Sage Publications. 
Raeve LD, Jansen NWH, van den Brandt PA, et al. (2009) Interpersonal conflicts at work as a predictor of selfreported health outcomes and ocupational mobility. Occup Environ Med 66: 16-22. doi: http://dx.doi.org/10.1136/oem.2007.037655.

Reneflot A and Mamelund S-E. (2012) The association between marital status and psychological well-being in Norway. Eur Soc Rev 28: 335-365. doi: http://dx.doi.org/10.1093/ esr/jcq069.

Romanov K, Appelberg K, Honkasalo M-L, et al. (1996) Recent interpersonal conflict at work and psychiatric morbidity: a prospective study of 15,530 employees aged 24-64. J Psychosom Res 40: 169-176. doi: http://dx.doi.org/10.1016/0022-3999(95)00577-3.

Sakurai K, Nakata A, Ikeda T, et al. (2014) Employment type, workplace interpersonal conflict, and insomnia: a cross-sectional study of 37,646 employees in Japan. Arch Environ Occup Health 69: 23-32. doi: http://dx.doi.org/10.1080/19338244.2012.713040.

Sandanger I, Moum T, Ingebrigtsen G, et al. (1999) The meaning and significance of caseness: the Hopkins Symptom Checklist-25 and the Composite International Diagnostic Interview. II. Soc Psychiatry Psychiatr Epidemiol 34: 53-59. doi: http://dx.doi.org/10.1007/ s001270050112.

Savin-Baden M and Major CH. (2013) Qualitative Research: The Essential Guide to Theory and Practice. London and New York: Routledge.

Tsuno K, Kawakami N, Inoue A, et al. (2009) Intragroup and intergroup conflict at work, psychological distress, and work engagement in a sample of employees in Japan. Ind Health 47: 640-648. doi: http://dx.doi.org/10.2486/indhealth.47.640.

Winokur A, Guthrie MB, Rickels K, et al. (1982) Extent of agreement between patient and physician ratings of emotional distress. Psychosomatics 23: 1135-1138, 1143, 1146. doi: http://dx.doi.org/10.1016/s0033-3182(82)73280-3. 